Original article

\title{
Large breast size as a risk factor for late adverse effects of breast radiotherapy: Is residual dose inhomogeneity, despite 3D treatment planning and delivery, the main explanation?
}

\author{
Christy Goldsmith ${ }^{\mathrm{a}, *}$, Joanne Haviland ${ }^{\mathrm{b}}$, Yat Tsang ${ }^{\mathrm{c}}$, Mark Sydenham ${ }^{\mathrm{b}}$, John Yarnold ${ }^{\mathrm{d}}$, \\ on behalf of the FAST Trialists' Group

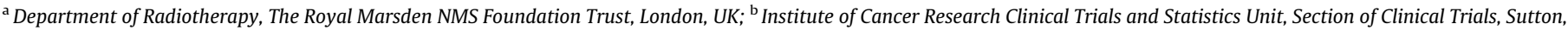 \\ UK; ' ${ }^{\mathrm{c}}$ Department of Medical Physics, Mount Vernon Cancer Centre, Northwood, UK; ${ }^{\mathrm{d}}$ Section of Academic Radiotherapy, The Royal Marsden NHS Foundation Trust, Sutton, UK
}

\section{A R T I C L E I N F O}

Article history:

Received 13 June 2010

Received in revised form 15 December 2010

Accepted 31 December 2010

Available online $\mathrm{xxxx}$

\section{Keywords:}

Radiotherapy

Breast size

Dosimetry

Late effects

\begin{abstract}
A B S T R A C T
Background and Purpose: Large breast size is associated with an increased risk of late adverse effects after breast conservation surgery and radiotherapy, even when 3D dosimetry is used. The purpose of this study is to test the hypothesis that residual dose inhomogeneity is sufficient to explain the association. Methods: Patients previously treated after breast conservation surgery with whole breast radiotherapy using 3D dosimetry and followed up in the UK FAST hypofractionation trial were selected for this analysis. The residual level of dose inhomogeneity across the whole breast treatment volume was used to test for association between residual dosimetry and post-treatment change in breast appearance at 2 years post-radiotherapy.

Results: At 2 years, 201/279 (72\%) of women had no change in photographic breast appearance, 61 (22\%) had mild change and 17 (6\%) had marked change. Breast size and dosimetry were both significantly associated with late effects in univariate analyses, but only breast size remained an independent significant risk factor for change in breast appearance when included in a multiple regression model together with other prognostic factors ( $p=0.006$ for trend).

Conclusion: Large-breasted women are more likely to suffer change in breast size and shape after whole breast radiotherapy delivered using 3D dosimetry, but residual dose inhomogeneity is insufficient to explain the association.
\end{abstract}

(C) 2011 Elsevier Ireland Ltd. All rights reserved. Radiotherapy and Oncology xxx (2011) xxx-xxx
There is retrospective evidence that breast size is a risk factor for late adverse effects following breast conservation surgery and adjuvant radiotherapy for early breast cancer [1-9]. Body mass index, correlated to breast size and brassiere size, has also been linked to the risk of acute skin reactions and to late cosmesis [10-12]. Not all studies are confirmatory, but the weight of evidence suggests that the association between the size of the breast and the risk of early and late adverse effects in the breast is real [13-16]. Analyses are based on assessments of late adverse effects made in clinic or from photographs, focusing on changes in breast size and shape, breast oedema and telangiectasia. Marked acute skin reactions in the inframammary fold of heavy-breasted patients may explain part of the association via an increased risk of consequential late effects.

* Corresponding author. Address: Department of Radiotherapy, Royal Marsden Hospital, Fulham Road, Chelsea, London SW3 6JJ, UK.

E-mail addresses: Christy.Goldsmith@rmh.nhs.uk, christyg@doctors.org.uk (C. Goldsmith).
Suboptimal dosimetry is thought to explain at least part of the association between breast size and risk of late normal tissue damage after radiotherapy delivered using $2 \mathrm{D}$ techniques $[1,2,4,6,17]$. A cause and effect relationship is supported by the 5-year results of a randomised trial $(N=306)$ comparing $2 \mathrm{D}$ and 3D radiation dosimetry [18], but not so far by the 2-year results of a larger confirmatory study [19]. Assuming dose distribution matters, it is not known if residual dose inhomogeneity in patients treated using 3D dosimetry explains a significant component of late adverse effects in large breasted women. Other factors associated with late adverse effects, including age [20], scar visibility, large excision volume, post-operative complications [12], smoking and adjuvant chemotherapy [11,21], appear unlikely to provide an adequate alternative explanation, since they are not all associated with breast size. Against this background, a retrospective analysis has been undertaken to test the hypothesis that residual dose inhomogeneity of whole breast radiotherapy delivered in conformity with ICRU recommendations (95-107\% of reference) [22,23] accounts for a significant part of the association between breast size and risk of late adverse effects.

0167-8140/\$ - see front matter @ 2011 Elsevier Ireland Ltd. All rights reserved. doi: $10.1016 /$ j.radonc. 2010.12 .012 


\section{Methods}

\section{Study population}

Patients were selected from those participating in the UK FAST hypofractionation trial, which randomised 915 patients between October 2004 and March 2007 to 25- and 5-fraction regimens of whole breast radiotherapy after primary tumour excision of early breast cancer [24]. Eligibility criteria included age $\geqslant 50$ years, invasive breast carcinoma, $<3 \mathrm{~cm}$ microscopic diameter, complete microscopic resection, negative axillary histology and written informed consent. Women were ineligible for trial entry if they required a tumour bed radiotherapy boost dose or neo-adjuvant/ adjuvant chemotherapy. Patients were randomised (1:1:1) between 50 Gy in 25 fractions of 2.0 Gy over 35 days (Control group), $30 \mathrm{~Gy}$ in five fractions of $6.0 \mathrm{~Gy}$ or $28.5 \mathrm{~Gy}$ in five fractions of $5.7 \mathrm{~Gy}$ treating once a week over 29 days (Test groups 1 and 2). Only a subset of the 18 radiotherapy centres participating in the FAST trial had compatible radiotherapy planning systems that allowed importation of data for joint analysis. Patients from the six centres with compatible planning systems were eligible for inclusion in the study.

\section{Virtual simulation and 3D dosimetry in the FAST trial}

Participating centres using 3D X-ray computer tomography (CT) imaging were included. Patients were imaged in the supine position using a recommended specialised breast board for immobilisation. Reproducibility of position was achieved by matching orthogonal laser beams with anterior and bilateral skin tattoos. The clinical target volume (CTV) was defined to include soft tissues of the whole breast down to the deep fascia, but not including underlying muscle and ribcage, nor overlying skin and excision scar. The inferior, medial and lateral limits of the breast were localised by inspection and palpation of the breast before $\mathrm{CT}$ images were collected. The superior border was defined in relation to the suprasternal notch. The planning target volume (PTV) included these limits to the CTV plus a $1 \mathrm{~cm}$ expansion in four radial directions. The radiotherapy plan for all patients was generated using two standard tangential fields with non-divergent posterior beam edges. The recommended maximum lung distance and maximum heart distance in the treatment volume was $<2$ and $<1 \mathrm{~cm}$, respectively. Cardiac shielding, where appropriate, was introduced using a multi-leaf collimator or other shielding technique.

The prescription point was half-way between the anterior lung surface and the skin surface on the perpendicular bisector of the posterior treatment beam edge. The FAST protocol followed guidelines of the International Commission on Radiation Units and Measurements (ICRU) reports 50 (1993) and 62 (1999) recommending dose variation throughout the treated volume between $-5 \%$ and $+7 \%$ [22,23]. If a standard wedged pair was insufficient, full dose compensation was performed using either missing tissue compensators based on outlines or portal images, or intensity modulated fields generated by forward or inverse planning. In practice, a forward-planned multi-leaf collimator (MLC) segment method was the dose compensation technique most commonly employed by participating centres. The body contours were obtained by CT and transferred to the planning system. Standard treatment fields were applied, and dose homogeneity was evaluated. Any hot spots of dose greater than $107 \%$ were minimised. A sagittal view through the breast was created with isodose lines greater than $107 \%$ displayed, see Fig. 1. A segment field was added using MLC to shield hot spots. If these were still present, an additional segment was used. The relative weightings of the segment fields tended to be very small (around 5\%). Six MV energy X-rays were recommended in the majority of patients, but for patient separations $>22 \mathrm{~cm}$, higher energies were considered to optimise dosimetry.

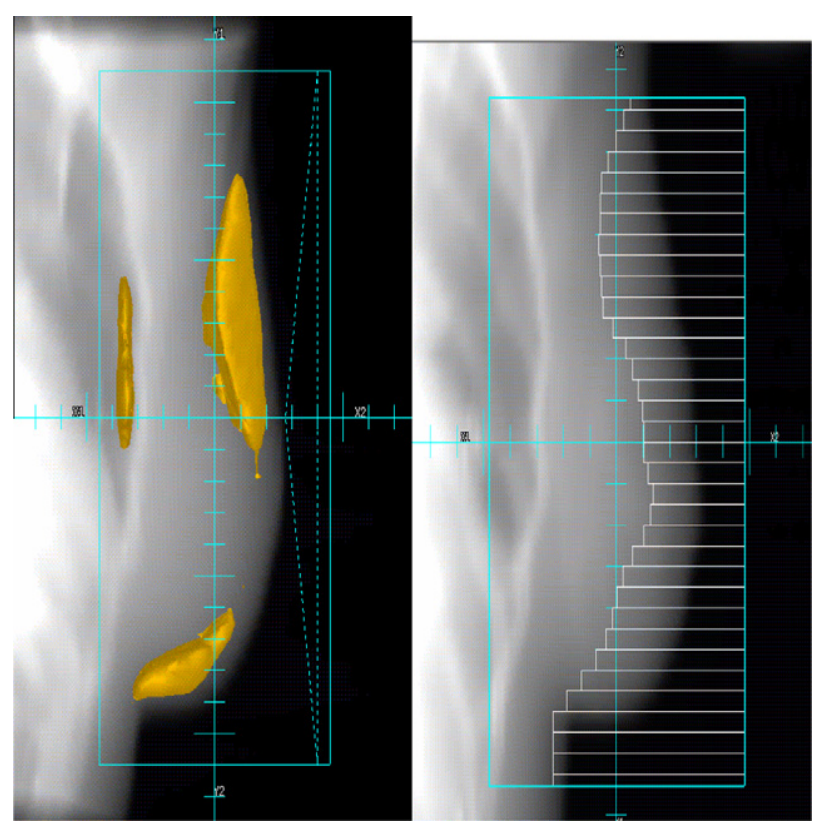

Fig. 1. Illustration of the use of multi-leaf collimators (MLCs) to shield hot-spots (orange zones).

Definition and assessment of late adverse radiation effects in the FAST trial

The primary endpoint of the FAST trial was change in photographic breast appearance at 2 and 5 years post-radiotherapy compared to post-surgical baseline photographs. Photographs (two frontal views of the chest, one with hands on hips and one with arms raised) were prospectively collected under standard conditions. Pairs of photographs (baseline and follow-up) were reviewed by observers blinded to treatment allocation and scored for change in breast appearance according to a three-point graded scale (no change/mild change/marked change) [25]. The START trial confirmed year two to be a valid time point for assessment, given a strong correlation between photographic scores at years two and five [26].

\section{Assessment of dose-volume histogram (DVH) data for current study}

Dosimetry data provided by FAST centres with compatible planning systems for central analysis for this current study were in the DICOMRT or RTOG format. It was not a mandatory trial requirement for participating FAST centres to outline whole breast volumes on CT scans, as already described. In order to carry out the dose-volume histogram (DVH) analysis required for this study, the entire dose cube of each radiotherapy plan was exported into a spreadsheet allowing the DVH data to be calculated for each isodose bin. DVH data were grouped into the following isodose bins: 50-90\%, 90-95\%, 95-97\%, 97-100\%, 100-103\%, 103-105\%, 105$107 \%,>107 \%$.

\section{Assessment of breast size for current study}

A measure of breast volume in cubic centimetres (cc) was estimated using radiotherapy planning X-ray CT images, but since the superior, inferior, medial and lateral limits of the CTV were defined by inspection and palpation, a breast CTV was not calculated. Instead, tissue lying within the $50 \%$ isodose line was selected as an appropriate surrogate measure, less dependent than the 95\% isodose line on the planning system and on the different X-ray energies selected for treatment. The $50 \%$ isodose line included 
non-target tissues, including ribcage, pectoral muscle and skin, that contribute to late adverse effects scored in the breast, and lung tissue which does not. This measure of breast volume was validated against photographic breast size scored clinically by consensus between three observers applying a three-point graded scale (small, medium, large) to images taken post-surgery and before radiotherapy under standard conditions, as previously described $[26,27]$.

\section{Statistical methods}

A parameter representing the proportion of breast volume receiving $>100 \%$ of prescribed dose was created from the dosevolume histogram data. Raw data for absolute breast volume (representing breast size) and percentage volume of breast tissue receiving $>100 \%$ of prescribed dose were summarised and tested using non-parametric methods (median, interquartile range, Kruskal-Wallis test) as the distributions were skewed. As very few patients had marked change in breast appearance, the mild and marked categories were combined for the analysis to define an endpoint of any change in photographic breast appearance. The $100 \%$ reference point did not represent an arbitrary cut-point for the analysis. Higher cut-points were considered, but these sampled a more limited part of the dose-volume distribution. It was assumed that late adverse effects would vary more in response to large partial volumes exposed to doses ranging from $101 \%$ to $107 \%$ than to very small partial volumes receiving $>107 \%$, for example. In the patients with any volumes receiving $>107 \%$, the median percentage volume of treated breast receiving dose $>107 \%$ was extremely small $(0.1 \%)$. This figure for partial breast volumes receiving $>107 \%$ of prescribed dose is consistent with previous reports in the literature [28]. The distribution of volume receiving $>100 \%$ dose was split into quartiles for the analysis assessing the effect of dosimetry on risk of late adverse effects. Logistic regression was used to assess the effect of breast size and dosimetry on the risk of any change in photographic breast appearance at 2 years. Each factor was first tested alone in a univariate model, and then both were included together to test whether the effects were independent of each other. Finally other factors associated with risk of late adverse effects (such as randomised radiotherapy fractionation regimen, surgical deficit, age, and adjuvant systemic therapy) were tested along with breast size and dosimetry in a multiple logistic regression analysis, and the final model retained those factors which were statistically significant.

\section{Results}

The FAST trial randomised 915 patients between October 2004 and March 2007 [24]. Although 16 out of 18 radiotherapy centres in the FAST trial used a CT planning system, only six centres had systems that allowed importation of data for joint analysis using Guiness software. (Royal Cornwall Hospital, Truro; The Royal Marsden Hospital, Sutton; Ipswich Hospital; Royal Shrewsbury Hospital; University Hospital of North Staffordshire, Stoke-onTrent and Beatson Oncology Centre, Glasgow). A total of 279 patients at these centres had baseline and 2-year photographs available for the analysis. The patient tumour and treatment characteristics of the study sample were representative of the FAST trial population as a whole $(n=915)$, see Table 1 . Age, time between surgery and randomisation, histological type, pathological tumour size, and tumour grade were comparable between the sample and the total FAST population. Adjuvant systemic therapy (endocrine treatment, usually tamoxifen $20 \mathrm{mg}$ OD for 5 years) and axillary node sampling were used more frequently in the study sample than the total FAST population.
Table 1

Patient characteristics of dosimetry study sample $(n=279)$ compared with overall distribution in FAST Trial $(n=915)$.

\begin{tabular}{|c|c|c|}
\hline & $\begin{array}{l}\text { Distribution in study } \\
\text { sample, } n(\%)\end{array}$ & $\begin{array}{l}\text { Overall distribution } \\
\text { in FAST trial }(\%)\end{array}$ \\
\hline \multicolumn{3}{|l|}{ Age (years) } \\
\hline $50-59$ & $90(32)$ & 36 \\
\hline $60-69$ & $135(48)$ & 48 \\
\hline $70-79$ & $47(17)$ & 14 \\
\hline $80-$ & $7(3)$ & 2 \\
\hline Mean (SD) [range] & $63.8(7.6)[50-88]$ & $62.9(7.2)[50-88]$ \\
\hline \multicolumn{3}{|c|}{ Time from surgery to randomisation (weeks) } \\
\hline $\begin{array}{l}\text { Median (interquartile range) } \\
\text { [range] }\end{array}$ & $\begin{array}{l}5.5(4.4-7.0)[2.4- \\
22.1]\end{array}$ & $\begin{array}{l}5.8(4.3-7.4)[0.4- \\
22.1]\end{array}$ \\
\hline \multicolumn{3}{|l|}{ Histological type } \\
\hline Ductal & $218(78)$ & 74 \\
\hline Lobular & $27(10)$ & 10 \\
\hline Other & $34(12)$ & 16 \\
\hline \multicolumn{3}{|l|}{ Axillary surgery } \\
\hline Sampling & $183(66)$ & 44 \\
\hline Clearance & $53(19)$ & 27 \\
\hline SNB $^{\mathrm{a}}$ with or without sampling & $34(12)$ & 25 \\
\hline Other & $9(3)$ & 4 \\
\hline \multicolumn{3}{|l|}{ Pathological tumour size $(\mathrm{cm})$} \\
\hline$<1$ & $83(30)$ & 28 \\
\hline $1-$ & $148(53)$ & 54 \\
\hline $2-$ & $48(17)$ & 18 \\
\hline Mean $(S D)$ [range] & $1.3(0.6)[0.05-4.0]$ & $1.4(0.7)[0.05-11.4]$ \\
\hline \multicolumn{3}{|l|}{ Tumour grade } \\
\hline 1 & $95(34)$ & 34 \\
\hline 2 & $159(57)$ & 55 \\
\hline 3 & $25(9)$ & 11 \\
\hline \multicolumn{3}{|l|}{ Adjuvant systemic therapy } \\
\hline Yes & $259(93)$ & 88 \\
\hline No & $20(7)$ & 12 \\
\hline
\end{tabular}

a $\mathrm{SNB}=$ sentinel node biopsy.

At 2 years post-randomisation, 201/279 (72\%) women had no change in photographic breast appearance, 61 (22\%) had mild change and 17 (6\%) had marked change. The validity of photographic scores of breast size was confirmed in comparisons with breast volume data from the radiotherapy planning systems, see Fig. 2. There was a statistically significant increase in measured breast volume across the three categories of breast size $(p<0.001)$, with a median of $778 \mathrm{cc}$ in the small group, $1114 \mathrm{cc}$ in medium and $1357 \mathrm{cc}$ in large categories. Photographic scores of breast size in 279 (100\%) patients were used for the analysis because data compatibility issues allowed absolute breast volume (cc) within the 50\% isodose envelope to be estimated for only $171(61 \%)$ women.

Breast size was significantly associated with volume receiving $>100 \%$ of the prescribed dose; the median (interquartile range) volumes receiving $>100 \%$ were $30.3 \%$ (22.7-37.3), 39.7\% (33.7$45.3)$ and $38.1 \%$ (28.8-47.0) for small, medium and large breast categories, respectively $(p<0.001)$. Rates of any (mild or marked) change in photographic breast appearance at 2 years were highest (50\%) in large-breasted women, 33\% in the medium-breasted category and $21 \%$ in small-breasted patients. This trend was statistically significant in univariate analysis $(p<0.001$ for trend; Table 2). Breast size remained a significant predictor when other significant risk factors (fractionation schedule and surgical deficit) and the dosimetry parameter were included in the multiple regression model ( $p=0.006$ for trend; Table 2 ). This confirmed breast size as an independent risk factor for radiation-induced change in breast appearance 2 years after radiotherapy using 3D dosimetry. Only $24 \%$ of patients received any dose $>107 \%$ of prescribed dose, and there was no significant association with occurrence of late effects (chi-squared $p=0.64$ ). 


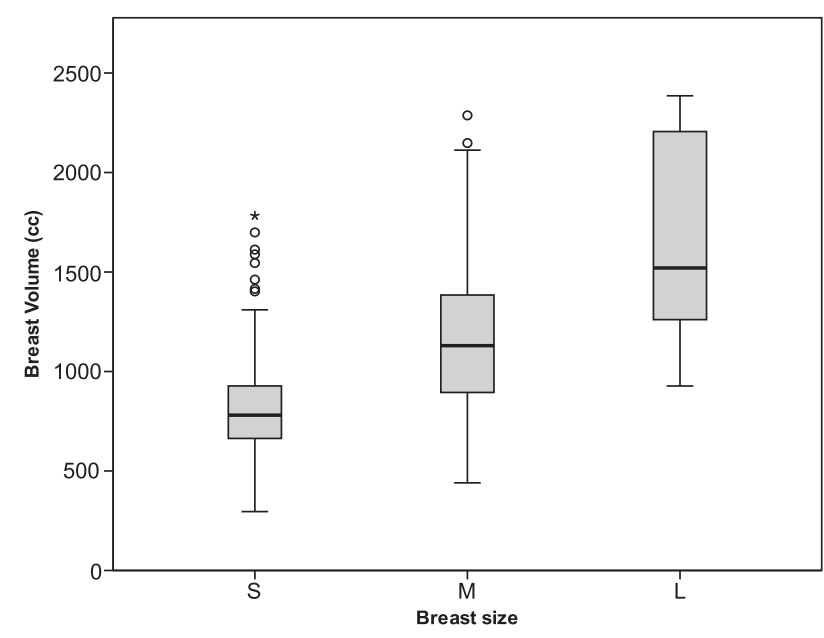

Fig. 2. Comparison of photographic assessment of breast size with actual measured breast volume. Median (interquartile range) breast volumes according to category of breast size (shown by bold lines and ends of boxes respectively): $778 \mathrm{cc}$ (660922) for small, $1114 \mathrm{cc}(846-1347)$ for medium and $1357 \mathrm{cc}$ (1193-1747) for large; $p<0.001$ for Kruskal-Wallis test.

The risk of any change in photographic breast appearance at 2 years increased with the proportion of breast volume receiving $>100 \%$ of the prescribed dose (22\% in the lowest and 39\% in the highest quartiles, respectively), which was significant in univariate analysis ( $p=0.009$ for trend; Table 2). However, when breast size and other significant risk factors (fractionation schedule and surgical deficit) were included in the multiple regression model, the effect of dosimetry was no longer significant $(p=0.136$ for trend; Table 2). This suggested that dose inhomogeneity, and other significant factors, left most of the effect of breast size on risk of late adverse effects unaccounted for, as indicated by odds ratios $(95 \% \mathrm{CI})$ for medium and large breasts compared with small of $1.58(0.83-$ 3.00 ) and 3.87 (1.53-9.80), respectively, see Table 2.

\section{Discussion}

Our data confirmed a significant association between breast size and dose inhomogeneity, in accordance with earlier literature $[1,2,4,6,17]$. It also confirmed breast size as a significant risk factor for change in photographic breast appearance, also consistent with a majority of published reports [2-9]. The effect of dose inhomogeneity on late adverse effects of 2D-planned breast radiotherapy has been tested in two randomised trials. Donovan et al. [29] randomised 306 patients requiring adjuvant whole breast radiotherapy to $2 \mathrm{D}$ radiotherapy using standard wedge compensators or 3D intensity modulated radiotherapy using multiple static fields. The 2D-planned patients were 1.7 times more likely to have a change in breast appearance at 5 years, judged by change in photographic breast appearance ( $p=0.008$ ), an effect not reproduced at 2 years follow-up in a second trial [19]. Against our expectations, the present analysis failed to confirm residual dose inhomogeneity in patients planned and treated using 3D dosimetry as an independent risk factor for radiation-induced change in breast appearance.

We consider it unlikely that a major effect of residual dose inhomogeneity on risk of late effects in large-breasted patients treated in accordance with IUCC guidelines has been missed. The measure of breast size, although based on a categorical scale applied to clinical photographs, correlated with absolute breast size measured from 3D X-ray CT images of the breast in a subset (61\%) of patients. Change in photographic appearance as an indicator of radiotherapy adverse effects has been well validated in randomised clinical trials conducted by our group, showing it to be sensitive to small $(<10 \%)$ differences in randomised total dose $[26,27]$. In the present study, $24 \%$ of patients received $>107 \%$ of prescribed dose to an average partial breast volume of only $0.44 \%$, so a correlation between patients receiving doses $>107 \%$ and probability of late effects could not be expected.

Although breast size and dosimetry were each significantly associated with late effects of breast radiotherapy on univariate analysis, dosimetry was no longer significant when included in a multivariate model including breast size. However, breast size remained an independent significant predictor of late effects when included in a multivariate model along with fractionation schedule, surgical deficit and dosimetry. The inference is that breast size, not dosimetry, is the dominant risk factor for late effects following 3D planned breast radiotherapy.

Late effects in this study were assessed by scoring photographic breast appearance, using the contralateral breast to control for age- and weight-related changes. The effects of radiotherapy most easily noticed on photographs are changes in size and shape, with breast shrinkage the commonest effect. In large- or heavy-breasted women, the major component of the breast by volume is adipose tissue $[30,31]$. This raises the possibility that adipose tissue in large- or heavy-breasted women is, in some way, more sensitive to the effects of radiotherapy. Whatever the mechanisms explaining why heavybreasted women are more likely to experience breast shrinkage and other late adverse effects, the challenge is to reduce treatment morbidity without negatively impacting on local control or survival rates. This report suggests that residual dose inhomogeneity in

Table 2

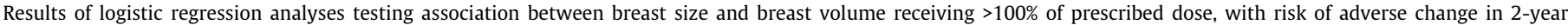
photographic breast appearance in 279 patients.

\begin{tabular}{|c|c|c|c|c|}
\hline & $\begin{array}{l}\text { Mild/marked change in breast } \\
\text { appearance at } 2 \text { years/total (\%) }\end{array}$ & $\begin{array}{l}\text { Crude OR }(95 \% \mathrm{CI}) \\
p \text {-value (trend) }\end{array}$ & $\begin{array}{l}\text { Adjusted } \mathrm{OR}^{\mathrm{a}}(95 \% \mathrm{CI}) \\
p \text {-value (trend) }\end{array}$ & $\begin{array}{l}\text { Adjusted } \mathrm{OR}^{\mathrm{b}}(95 \% \mathrm{CI}), \\
p \text {-value (trend) }\end{array}$ \\
\hline & & $p<0.001$ & $p=0.012$ & $p=0.006$ \\
\hline \multicolumn{5}{|c|}{ Breast size } \\
\hline Small & $33 / 155(21.3)$ & 1 & 1 & 1 \\
\hline Medium & $32 / 98(32.7)$ & $2.04(1.40-2.30)$ & $1.49(0.81-2.74)$ & $1.58(0.83-3.00)$ \\
\hline \multirow[t]{2}{*}{ Large } & $13 / 26(50.0)$ & $4.13(2.43-7.01)$ & $3.21(1.32-7.80)$ & $3.87(1.53-9.80)$ \\
\hline & & $p=0.009$ & $p=0.072$ & $p=0.136$ \\
\hline \multicolumn{5}{|c|}{$\%$ Volume receiving $>100 \%$ dose $e^{c}$} \\
\hline$<27$ & $16 / 74(21.6)$ & 1 & 1 & 1 \\
\hline $27-34$ & $15 / 71(21.1)$ & $0.97(0.44-2.15)$ & $0.85(0.38-1.93)$ & $0.68(0.29-1.60)$ \\
\hline $35-41$ & $19 / 63(30.2)$ & $1.56(0.72-3.39)$ & $1.34(0.60-2.98)$ & $1.18(0.52-2.69)$ \\
\hline$>42$ & $28 / 71(39.4)$ & $2.36(1.14-4.90)$ & $1.82(0.83-3.98)$ & $1.62(0.72-3.64)$ \\
\hline
\end{tabular}

a Odds ratio adjusted for breast size and \% volume receiving $>100 \%$ dose.

b Odds ratio adjusted for breast size, \% volume receiving $>100 \%$ dose, fractionation schedule and surgical deficit.

c Quartiles used to define categories. 
patients treated with optimal dosimetry is not able to negate the risk factor of large breast size. If confirmed by other studies, one implication would be to consider a small dose reduction to compensate for the enhanced risk of late adverse effects. A $10 \%$ reduction in total dose would reduce the absolute risk of late adverse effects at 2 years by $20 \%$, assuming a $\gamma 50$ of 2 to describe the slope of the dose response curve at the $50 \%$ level of effect. In the setting of adjuvant breast radiotherapy, this dose reduction to whole breast would be associated with a $\leqslant 1 \%$ loss of local tumour control, assuming $\gamma 95$ of 0.2 at the $95 \%$ level of local tumour control $[27,32]$.

\section{Conclusion}

Large- or heavy-breasted women are more likely than others to suffer change in breast size and shape after radiotherapy, even when 3D dosimetry has been optimised.

\section{Acknowledgements}

We acknowledge the FAST Trialists' Group, which in addition to the co-authors John Yarnold, Joanne Haviland and Mark Sydenham includes: Rajiv Agrawal, Royal Shrewsbury Hospital, Shrewsbury; Abdulla Alhasso, Beatson Oncology Centre, Glasgow; Jane Barrett, Royal Berkshire Hospital, Reading; Peter Barrett-Lee, Velindre Hospital, Cardiff; Judith Bliss, Institute of Cancer Research Clinical Trials and Statistics Unit, Section of Clinical Trials, Sutton; David Bloomfield, Royal Sussex County Hospital, Brighton; Joanna Bowen, Cheltenham General Hospital, Cheltenham; Murray Brunt, University Hospital of North Staffordshire, Stoke-on-Trent; Ellen Donovan, The Royal Marsden NHS Foundation Trust, Sutton; Andy Goodman, Royal Devon \& Exeter NHS Trust, Exeter; Marianne Illsley, Royal Surrey County Hospital, Guildford; Assem Rostom, The Royal Marsden NHS Foundation Trust, Sutton; Elizabeth Sherwin, Ipswich Hospital, Ipswich; Alan Stewart, Christie Hospital, Manchester and Duncan Wheatley, Royal Cornwall Hospital, Truro.

\section{References}

[1] Moody AM, Mayles WP, Bliss JM, et al. The influence of breast size on late radiation effects and association with radiotherapy dose inhomogeneity. Radiother Oncol 1994;33:106-12.

[2] Brierley JD, Paterson IC, Lallemand RC, Rostom AY. The influence of breast size on late radiation reaction following excision and radiotherapy for early breast cancer. Clin Oncol (R Coll Radiol) 1991;3:6-9.

[3] Clarke D, Martinez A, Cox RS. Analysis of cosmetic results and complications in patients with stage I and II breast cancer treated by biopsy and irradiation. Int J Radiat Oncol Biol Phys 1983;9:1807-13.

[4] Gray JR, McCormick B, Cox L, Yahalom J. Primary breast irradiation in largebreasted or heavy women: analysis of cosmetic outcome. Int J Radiat Oncol Biol Phys 1991;21:347-54.

[5] Harris JR, Levene MB, Svensson G, Hellman S. Analysis of cosmetic results following primary radiation therapy for stages I and II carcinoma of the breast. Int J Radiat Oncol Biol Phys 1979;5:257-61.

[6] Liauw SH, Sanfilippo LJ, Santoro E. Breast size versus cosmesis and local control in stages I and II breast carcinoma. N J Med 1987;84:706-10.

[7] Ray GR, Fish VJ, Lee RH, et al. Biopsy and definitive radiation therapy in stages I and II carcinoma of the female breast. Int J Radiat Oncol Biol Phys 1983;9:23-8.

[8] Tsouskas LI, Fentiman IS. Breast compliance: a new method for evaluation of cosmetic outcome after conservative treatment of early breast cancer. Breast Cancer Res Treat 1990;15:185-90.

[9] Pezner RD, Patterson MP, Hill LR, Desai KR, Vora N, Lipsett JA. Breast edema in patients treated conservatively for stage I and II breast cancer. Int J Radiat Oncol Biol Phys 1985;11:1765-8.
[10] Twardella D, Popanda O, Helmbold I, et al. Personal characteristics, therapy modalities and individual DNA repair capacity as predictive factors of acute skin toxicity in an unselected cohort of breast cancer patients receiving radiotherapy. Radiother Oncol 2003;69:145-53.

[11] Fernando IN, Ford HT, Powles TJ, et al. Factors affecting acute skin toxicity in patients having breast irradiation after conservative surgery: a prospective study of treatment practice at the Royal Marsden Hospital. Clin Oncol (R Coll Radiol) 1996;8:226-33.

[12] Vrieling C, Collette L, Fourquet A, et al. The influence of patient, tumor and treatment factors on the cosmetic results after breast-conserving therapy in the EORTC 'boost vs. no boost' trial. EORTC Radiotherapy and Breast Cancer Cooperative Groups. Radiother Oncol 2000;55:219-32.

[13] Bulman AS, Cassoni AM, Ellis H. The functional results following primary treatment of breast cancer with breast conservation. Eur J Surg Oncol 1985;11:247-9.

[14] Habibollahi F, Mayles HM, Mayles WP, et al. Assessment of skin dose and its relation to cosmesis in the conservative treatment of early breast cancer. Int J Radiat Oncol Biol Phys 1988;14:291-6.

[15] Hamilton CS, Nield JM, Adler GF, Clingan PR. Breast appearance and function after breast conserving surgery and radiotherapy. Acta Oncol 1990;29:291-5.

[16] Dewar JA, Benhamou S, Benhamou E, et al. Cosmetic results following lumpectomy, axillary dissection and radiotherapy for small breast cancers. Radiother Oncol 1988;12:273-80.

[17] Neal AJ, Torr M, Helyer S, Yarnold JR. Correlation of breast dose heterogeneity with breast size using 3D CT planning and dose-volume histograms. Radiother Oncol 1995;34:210-8. 82:254-64.

[18] Donovan E, Bleakley N, Denholm E, et al. Randomised trial of standard 2D radiotherapy (RT) versus intensity modulated radiotherapy (IMRT) in patients prescribed breast radiotherapy. Radiother Oncol 2007;82:254-64.

[19] Coles CE. A randomised controlled trial of forward-planned IMRT for early breast cancer: interim results at 2 years follow-up. Session VI, Abstract 71, San Antonio Breast Cancer Conference; 2009.

[20] Lilla C, Ambrosone CB, Kropp S, et al. Predictive factors for late normal tissue complications following radiotherapy for breast cancer. Breast Cancer Res Treat 2007; 106:143-50.

[21] Borger JH, Kemperman H, Smitt HS, et al. Dose and volume effects on fibrosis after breast conservation therapy. Int J Radiat Oncol Biol Phys 1994;30:1073-81.

[22] ICRU, Prescribing, recording and reporting photon beam therapy. International Commission on Radiation Units and Measurements (ICRU); 1993.

[23] ICRU, Prescribing, recording and reporting photon beam therapy. Interantional Commission on Radiation Units and Measurements (ICRU); 1999.

[24] Brunt AM, Sydenham M, Bliss J, et al. A 5-fraction regimen of adjuvant radiotherapy for women with early breast cancer: first analysis of the randomised UK FAST trial (ISRCTN62488883, CRUKE/04/015). EJC Supplements. Presidential sessions late breaking and best of ECCO 15-ESMO 34 Abstracts 2009;7:2.

[25] Haviland JS, Ashton A, Broad B, et al. Evaluation of a method for grading late photographic change in breast appearance after radiotherapy for early breast cancer. Clin Oncol (R Coll Radiol) 2008;20:497-501.

[26] Bentzen SM, Agrawal RK, Aird EG, et al. The UK Standardisation of Breast Radiotherapy (START) Trial A of radiotherapy hypofractionation for treatment of early breast cancer: a randomised trial. Lancet Oncol 2008;9:331-41.

[27] Yarnold J, Ashton A, Bliss J, et al. Fractionation sensitivity and dose response of late adverse effects in the breast after radiotherapy for early breast cancer: long-term results of a randomised trial. Radiother Oncol 2005;75:9-17.

[28] Barnett GC, Wilkinson J, Moody AM, et al. A randomised controlled trial of forward-planned radiotherapy (IMRT) for early breast cancer: baseline characteristics and dosimetry results. Radiother Oncol 2009;92:34-41.

[29] Yarnold JR, Donovan E, Bleackley N, et al. Randomised trial of standard 2D radiotherapy versus 3D intensity modulated radiotherapy (IMRT) in patients prescribed breast radiotherapy. Radiother Oncol 2002;64:S25.

[30] Riza E, Remoundos DD, Bakali E, Karadedou-Zafiriadou E, Linos D, Linos A. Anthropometric characteristics and mammographic parenchymal patterns in post-menopausal women: a population-based study in Northern Greece. Cancer Causes Control 2009;20:181-91.

[31] Caire-Juvera G, Arendell LA, Maskarinec G, Thomson CA, Chen Z. Associations between mammographic density and body composition in Hispanic and non-Hispanic white women by menopause status. Menopause 2008;15:319-25.

[32] Owen JR, Ashton A, Bliss JM, et al. Effect of radiotherapy fraction size on tumour control in patients with early-stage breast cancer after local tumour excision: long-term results of a randomised trial. Lancet Oncol 2006;7:467-71. 